\title{
Análisis de la Educación Intercultural en la enseñanza superior. El caso de la Comunidad Autónoma de Andalucía
}

\section{Analysis of Intercultural Education in higher education. The case of the Autonomous Community of Andalucía}

\author{
José Antonio Ruiz-Rodríguez ${ }^{1}$, Manuel Delgado-García ${ }^{2}$ \\ ${ }^{1}$ Universidad de Huelva. joseantonio.ruiz@dedu.uhu.es \\ ${ }^{2}$ Universidad de Huelva. manuel.delgado@dedu.uhu.es
}

Recibido: $15 / 6 / 2021$

Aceptado: 25/10/2021

Copyright (C)

Facultad de CC. de la Educación y Deporte.

Universidad de Vigo

\section{Dirección de contacto:}

José Antonio Ruiz Rodríguez

Facultad de Educación, Psicología y Ciencias del Deporte

Universidad de Huelva

Campus de "El Carmen"

Avenida de las Fuerzas Armadas, s/n

21007 Huelva

\begin{abstract}
Resumen
El fenómeno migratorio y la interculturalidad son realidades sociales atendidas desde las instituciones educativas a diferentes niveles. En los últimos años, los estudios centrados en este campo de conocimiento se han intensificado y, como consecuencia, este trabajo pretende dar a conocer cómo se integra la formación en Educación Intercultural en las titulaciones universitarias oficiales andaluzas. Desde una perspectiva de análisis cualitativo, apoyado en el análisis documental (planes de estudios y guías docentes), se aborda comparativamente la realidad de 4 universidades. Los resultados reflejan diferencias en el tratamiento de la Educación Intercultural y se infiere cierta relación con el saldo migratorio. Finalmente, se realiza una propuesta de mejora que ayude a optimizar la formación universitaria en Educación Intercultural.
\end{abstract}

\section{Palabras clave}

Enseñanza Superior, Educación Intercultural, Análisis Documental

\section{Abstract}

The migratory phenomenon and interculturality are social realities attended by educational institutions at different levels. In recent years, studies focused on this field of knowledge have intensified and, as a consequence, this work aims to show how training in Intercultural Education is integrated into official Andalusian university degrees. From a qualitative analysis perspective, supported by documentary analysis (study plans and teaching guides), the reality of 4 universities is comparatively approached. The results reflect differences in the treatment of Intercultural Education and a certain relationship is inferred with the migratory balance. Finally, an improvement proposal is made to help optimize university training in Intercultural Education. 


\section{Key Words}

Higher Education, Intercultural Education, Documentary Analysis

\section{INTRODUCCIÓN}

"Diferentes formas, ideas, sonidos y olores culturales que se han desprendido de su lugar originario, se ofrecen en un hiperespacio sin fronteras (...). Ni el trans, ni el multi ni el inter, sino el hiper caracteriza la constitución cultural de hoy” (Han, 2018, pp. 83-84).

Con estas palabras, este autor hace referencia al fenómeno de la interculturalidad en la actualidad, pasando de una superación de la transculturalidad (paso de una cultura a otra) a la hiperculturalidad.

En este sentido, el tema de la migración existe en cualquier época, alcanzando un nivel global en todas las esferas de la vida humana. La literatura científica existente ha demostrado la trascendencia de investigar los factores que inciden en los movimientos migratorios y, con este trabajo, se pretende contribuir a profundizar en el análisis de dicho fenómeno desde el ámbito académico.

Esta investigación se fundamenta en visualizar la controversia existente entre las migraciones en Andalucía y los estudios existentes en las titulaciones oficiales de las Universidades Andaluzas. Como punto de partida, tomando en consideración las Universidades de Andalucía y las temáticas desarrolladas en los distintos ámbitos de conocimiento, se va a determinar una forma de catalogar la complejidad de temáticas encontradas, sus trascendencias, su distribución y su finalidad. Por tanto, la continua incorporación en las titulaciones oficiales de alumnado de diferentes culturas, arraigado por el fenómeno de las migraciones en el territorio español, da lugar a que en las instituciones educativas prolifere este tipo de alumnado y que se entremezclen entre sí, enriqueciéndonos en todo momento con este auge de la interculturalidad. Así lo corrobora el Instituto de Estadística y Cartografía de Andalucía (2020), cuando determina que en las últimas décadas se han incorporado al sistema educativo español multitud de alumnos y alumnas inmigrantes y, por tanto, el aumento de la interculturalidad ha desencadenado el incremento de formación específica en los todos los sectores de la comunidad educativa.

Muchos autores han venido reflejando esta preocupación desde hace años, tales como Popkewitz et al. (1986), quienes consideraban el problema de las desigualdades sociales como foco de las investigaciones y las políticas educativas, lo que obligó a un crecimiento de reformas de gran alcance; Puig (1995, p.75) reflexionaba de la siguiente manera: "ya nunca nuestra mirada podrá captar un paisaje monocultural. La diversidad de modos de vida y de culturas será para siempre el escenario de nuestra existencia"; Grañeras et al. (1998), reseñaban que, con la presencia masiva de inmigrantes en nuestras aulas, a partir de los años 90, aumentaba el interés y el número de investigaciones sobre Educación Intercultural en España.

En este sentido, Grañeras et al. (1998, citados en González-Falcón, 2006, p. 96), apuntan: 
“(...) que el aumento masivo del flujo de inmigrantes en los últimos años, ha supuesto para la escuela un reto difícilmente abordable con carácter inmediato por la falta de recursos, pero, sobre todo, por la falta de una formación específica del profesorado”.

En cuanto a la relación de este flujo migratorio con los centros educativos, Bueno (2008, p. 64), reseñaba que "la escuela es el instrumento fundamental para esta labor de integración, además de ser la escuela la principal facilitadora de este tránsito de una cultura a otra"; Etxeberría y Elósegui (2010), en relación al proceso de integración de los alumnos y las alumnas inmigrantes en nuestro sistema, comentaban que existen una serie de problemas y obstáculos, como la creciente concentración de este alumnado en centros públicos, las dificultades en el aprendizaje de la lengua o las lenguas de acogida, la pérdida de la lengua de origen, un rendimiento inferior a los nativos en pruebas estandarizadas, cierto rechazo social, etc.

Como se puede apreciar, a lo largo de los años, la interculturalidad en las titulaciones oficiales se ha ido visualizando a través de la literatura científica. Basándose en ello, en este estudio se profundiza en los fundamentos básicos de la Educación Intercultural en las titulaciones oficiales, unos principios que giran en torno a los siguientes ámbitos de conocimiento (Figura 1):

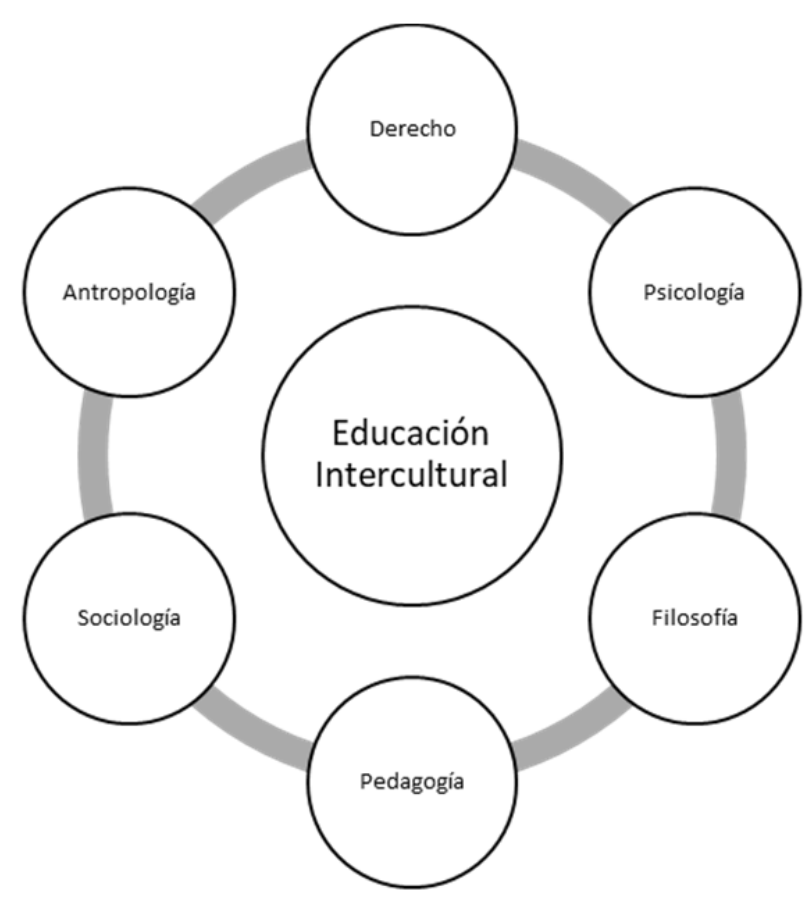

Figura 1. Ámbitos de conocimiento asociados a la Educación Intercultural

\subsection{Antecedentes}

Es imprescindible comenzar con una breve aproximación al pensamiento crítico de la mano de Soltonovich (2012), quien fundamenta que la teoría social "tradicional" trata de identificar en la organización social los elementos que atentan contra el funcionamiento de una sociedad "normal", atendiendo a las tensiones de la estructura social que determinarán las mutaciones históricas, unas relaciones jerarquizadas que generan conflicto, y que, por consiguiente, dan lugar a dos ejes centrales desde el prisma del pensamiento crítico: la visibilidad sobre las relaciones de poder y el conflicto 
como un elemento inherente a nuestra dinámica social; mientras que en la teoría tradicional el foco se encuentra en el consenso.

Para Horkheimer (2000, p. 223) esta teoría se define como "la acumulación del saber en forma tal, que éste se vuelva utilizable para caracterizar los hechos de la manera más acabada posible"; a su vez, en él se localizan tanto el "saber formulado conceptualmente, como aquella situación objetiva que debe ser incluida en aquel, y este acto de subsumir, de establecer la relación entre la simple percepción o comprobación del hecho y la estructura conceptual de nuestro saber (p.223)".

En palabras de Herrera (2005, p. 50) pensar es "pensar de otro modo, problematizar la realidad, identificar lo que en ella hay de problemático. Esta forma de pensamiento requiere, por tanto, fundar nuevas formas de acercarse tanto teórica como prácticamente al mundo". De este modo, una teoría es crítica si se focaliza en comprender aquellas situaciones discriminatorias que actúan como la base sistémica o estructural que acaban por generarlas (Gallardo, 2016).

Es evidente que se necesita avanzar en una concepción de los derechos no coloniales, e incluso anti-imperialista, exigiendo el reconocimiento de la diversidad cultural y denunciando la absolutización de aquellas formas de vida particulares con poder suficiente para imponerse en la dinámica histórica. Según Salvat (2001, p.31) “es necesario reconocer la universalidad de los derechos humanos no como punto de partida, como hecho dado, sino como meta posible, como horizonte hacia el cual caminar a través del diálogo intercultural". Se trata de dar valía a un conjunto de normas que ayuden a viabilizar un Derecho Internacional de los Derechos Humanos, donde cada una de estas normas posea expresiones diferenciadas en lo cultural o lo jurídico, en vez de un objetivo claramente homogenizador que poco tiene que ver con la finalidad hacia la que se avanza; es aquí donde el diálogo intercultural adquirirá el mayor protagonismo para poder contribuir a que aquellos valores, sentidos, normas, etc. se intercambien desde el respeto y la cordialidad.

Por tanto, para entender esta migración es indudable conocer antes los fundamentos básicos que se relacionan con el propio concepto, tales como la cultura, la diversidad cultural, el pluralismo cultural, la identidad cultural, el interculturalismo, el etnocentrismo, el relativismo cultural, la interculturalidad y la multiculturalidad.

En primer lugar, Díaz de Rada (2010, p. 247) nos habla del término de cultura, “como un atributo de la acción de las personas, que no está acotada en las fronteras de ningún grupo humano, una cultura no es independiente de otras culturas, ni puede serlo”. Por tanto, siguiendo a García Llamas (2005) aceptaríamos que la cultura es un aspecto que se comparte y vive con intensidad en un grupo social determinado, incluyendo sus propios valores, hábitos, experiencias, percepción y símbolos sociales o contextuales, etc., o lo que es lo mismo, habría que aceptar que no es posible formular una definición en un mismo sentido y forma, pues existen condicionantes contextuales que la caracterizarán y modelarán. En definitiva, el concepto de cultura estará condicionado por el entorno en el cual se desarrolla.

Grosso modo, podría decirse que son las personas las que hacen la cultura, apareciendo el término de diversidad cultural, entendido como aquella variedad de grupos, colectivos y culturas que producen un intercambio cultural de todos los grupos (gitanos, europeos, etc.) y por todos los agentes que intervienen (medios de comunicación, escuela, empresa, etc.). 
Otro de los conceptos vinculado a la migración es el de pluralismo cultural. Este “enfoque o manera de entender la educación multicultural surge de la no aceptación, por parte de las minorías étnicas, de las prácticas de aculturación y asimilación a las que se encuentran sometidas en el contacto con las culturas mayoritarias" (García et al., 2004, p. 87). A raíz de estos, emergen otros dos conceptos como son el multiculturalismo y el interculturalismo que están claramente unidos al primero. Cabe puntualizar que en el ámbito anglosajón predomina el término multiculturalismo, en cambio, el contexto europeo se decanta por el término de interculturalismo. En relación a este último, García Llamas (2005), hace hincapié en el "interculturalismo" como configuración de "una forma de intervención que hace especial hincapié en el valor que las relaciones e interacciones tienen para la convivencia de las culturas dentro de un mismo espacio geográfico y social" (p. 91).

En cambio, otros autores (Aguado, 1991; Molina, 1994; Carbonell, 1995, citados en González-Falcón, 2003, p. 74) coinciden en diferenciar multiculturalismo de interculturalismo, señalando que "el primero alude a la existencia de varias culturas en las mismas coordenadas espacio-temporales $\mathrm{y}$, el segundo, hace referencia a la interacción y mestizaje que se produce en el seno de tales comunidades".

Por otro lado, Hidalgo (2005, p. 80) nos habla de la palabra etnocentrismo como "el sentimiento o creencia que tienen las distintas personas de estar en posesión de la mejor cultura de entre las existentes en el mundo". Esto significa que la cultura de origen, la verdadera, es una forma de enjuiciar el resto de las culturas. Esta misma autora hace referencia al relativismo cultural entendido como "la atracción por las creencias, actitudes, valores, etc. de las otras culturas descubiertas y que, por tanto, todos los aspectos de la misma tienen sentido dentro de esta comunidad, incluso los aspectos que atentan contra los derechos humanos (p. 81)".

En cuanto al concepto de identidad cultural, Touriñán (2005) ayuda a definirlo a través de la relación entre el individuo y la sociedad, en concreto, a través de su concepción como parte de la realidad subjetiva. Se trata de un fenómeno que contribuye a definir la manera en que una persona se muestra, pertenece a un colectivo o se desenvuelve en el mundo (intervienen así la cultura, la tradición y la lengua). Todos los rasgos que caracterizan a una cultura serán los elementos que construyan la identidad de la persona y, por lo tanto, fortalecer la identidad cultural propia es requisito necesario en la práctica de la interculturalidad, dado que se entiende como los cimientos para establecer y consolidar relaciones interculturales duraderas y estables.

En resumen, Lozano (2005, p. 28) define la interculturalidad como "un proceso de interrelación que parte de la reflexión del reconocimiento de la diversidad y del respeto a las diferencias".

Por su parte, Giménez (2003) habla del término de interculturalidad como:

“la simple descripción de las relaciones interétnicas, interreligiosas o interlingüísticas; y el interculturalismo, dentro del plano normativo, como posición y propuesta de cómo deberían ser esas relaciones interculturales. Así, hace hincapié en la necesidad de intercambiar los planteamientos multiculturalistas por los interculturalistas, que no sólo adoptan la postura multicultural” (p. 5).

Así pues, la interculturalidad debe ser entendida como:

"un designio y propuesta de sociedad, como proyecto político, social, epistémico y ético dirigido a la transformación estructural y socio-histórica, asentado en la construcción entre todos de una sociedad radicalmente distinta. Una transformación y construcción que no 
quedan en el enunciado, el discurso o la pura imaginación; por el contrario, requieren de un accionar en cada instancia social, política, educativa y humana” (Walsh, 2007, pp. 4-5).

Una vez entendidos todos estos conceptos, es posible aclarar en qué consiste la migración. Esta terminología proviene de la conjunción del término de emigración, como "acto de salir de un Estado con el propósito de asentarse en otro" (OIM, 2006, p. 23), y el de inmigración, como "proceso por el cual las personas no nacionales ingresan en un país con el fin de establecerse en él" (OIM, 2006, p. 32). Por tanto, la migración sería un "movimiento de población hacia el territorio de otro Estado o dentro del mismo, que abarca todo movimiento de personas sea cual fuere su tamaño, su composición o sus causas; incluye migración de refugiados, personas desplazadas, personas desarraigadas, o migrantes económicos" (OIM, 2006, p. 38). Basándose en esto, se procedió al estudio de la Educación Intercultural como un eje donde deben tenerse en cuenta la inmigración y la emigración para sopesar el saldo migratorio.

\subsection{Estado del arte}

En la revisión de la literatura asociada al tema de investigación que se plantea en este artículo, Di Caudo-Villoslada (2020) nos habla del estudiante intercultural como vehículo para lograr que la interculturalidad recale dentro del ámbito estatal, fomentando la inserción en las titulaciones educativas oficiales de la Educación Intercultural.

En otros estudios, como el de Gil-Madrona et al. (2016) relativo a las creencias de los estudiantes sobre la multiculturalidad y el desarrollo de la competencia intercultural en la Universidad de Castilla-La Mancha (España), se reseñaba que el plan de estudios de Educación Infantil no mejoraba la interculturalidad, sino que la ocultaba. Hecho que Carrasco y Coronel (2017) afirmaron cuando mostraron en su investigación que la competencia intercultural se debe incluir en la formación permanente del profesorado.

González-González et al. (2012) analizaron la formación de los educadores profesionales en competencias interculturales (en las titulaciones pedagógicas de las Universidades de Burgos y Córdoba), proponiendo una secuenciación formativa en los profesionales. Por otro lado, Cala et al. (2018), en su investigación sobre las actitudes hacia los refugiados en los futuros profesores de los últimos años de Magisterio del sureste español, demostraron que estos sectores sociales son los menos afectados y reseñan la importancia de medidas urgentes en programas de formación intercultural. De esta manera, también propone Bernabé (2016), en las asignaturas de Música del Grado de Educación Infantil, una modificación en los currículos superiores en materia de interculturalidad.

En otra investigación, Fernández-Agüero y Garrote (2019) resaltan que el futuro profesorado muestra una identidad intercultural primitiva, tendente a evitar la intervención y con desconocimiento intercultural. Peñalva y Leiva (2019), en su investigación en los Grados de Magisterio en Educación Infantil, Primaria y Pedagogía de las Universidades de Málaga y Pública de Navarra, reflejan pocas actitudes positivas hacia la interculturalidad $\mathrm{y}$, al mismo tiempo, se solicita mayor formación en las diferentes capacidades que configuran la competencia intercultural. Espinoza et al. (2019) realizaron su estudio en la Universidad Técnica de Machala en Ecuador, donde observaron que para el alumnado que estudia la Educación Básica, se evidencia que la 
dimensión intercultural carece de espacio en el currículum, y se alude a mejorar la formación en Educación Intercultural.

Como solución a esta problemática, Sáez-López et al. (2016), llevaron a cabo una experiencia en relación al proceso de codificación para crear presentaciones multimedia en un contexto intercultural sobre los conceptos, actitudes y prácticas en tres importantes universidades de Japón, México y España.

Louzao (2019, p. 45) considera una serie de situaciones actuales que determinan los retos sobre la Educación Intercultural, tales como:

"la situación socio-política actual, con el auge reactivado de políticas racistas y xenófobas en Europa, la mala gestión de la crisis de los refugiados y el rechazo de una parte de la ciudadanía a su acogida; el debate emergente de los nacionalismos; el escaso éxito de las políticas de integración de las segundas generaciones de extranjeros en algunos países europeos, con los consecuentes disturbios sociales aparecidos, los recientes disturbios raciales en Madrid...”

Tomando en consideración el estado del arte descrito, este estudio centra el interés en las realidades que envuelven a los planes de estudios oficiales de las instituciones de enseñanza superior.

\section{OBJETIVOS}

La respuesta al estado del arte planteado se ofrece mediante la formulación de dos objetivos generales, con los que se trata de otorgar visibilidad al fenómeno objeto de estudio:

a) Dar a conocer cómo se integra la formación en Educación Intercultural en el seno de las guías docentes correspondientes a las titulaciones de Grados y Másteres Oficiales de las diferentes universidades andaluzas.

b) Exponer algunas propuestas que inviten a mejorar el proceso de inclusión de la Educación Intercultural en el currículo universitario.

\section{METODOLOGÍA}

Tomando como referencia los postulados asociados al paradigma interpretativo, se procede al diseño de una investigación de tipo cualitativo -no interactiva o analítica-, con una finalidad descriptiva y comparativa. Se emplea el análisis documental como estrategia básica para la indagación en el fenómeno de estudio. En este tipo de investigaciones, los documentos suponen la fuente principal de la información y, como investigadores, se ofrece una interpretación de ésta para determinar los significados que subyacen a las prácticas educativas actuales en el contexto de la Educación Superior y próximas al objeto de estudio (McMillan y Schumacher, 2005).

\subsection{Muestra}

Este trabajo parte de un universo más amplio que comprende un análisis de todos los planes de estudios y guías académicas de las titulaciones afines al ámbito de las Ciencias Sociales y Humanas en Universidades Públicas Andaluzas, para determinar la mención de la Educación Intercultural durante el curso académico 2019/2020 (Tabla 1). 


\begin{tabular}{|c|c|c|c|c|c|c|}
\hline 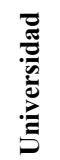 & $\begin{array}{c}\mathrm{N}^{0} \text { de } \\
\text { títulos por } \\
\text { nivel } \\
\text { educativo }\end{array}$ & $\begin{array}{c}\text { Con mención } \\
\text { a la } \\
\text { Educación } \\
\text { Intercultural }\end{array}$ & $\%$ & Asignaturas & $\begin{array}{c}\text { Con mención } \\
\text { a la } \\
\text { Educación } \\
\text { Intercultural }\end{array}$ & $\%$ \\
\hline \multirow{10}{*}{ } & \multirow{3}{*}{$\begin{array}{c}(34) \\
\text { Grados }\end{array}$} & \multirow{3}{*}{$\begin{array}{c}(3) \\
\text { Grados }\end{array}$} & \multirow{3}{*}{$8,8 \%$} & (37) Educación Infantil & 2 & $5,4 \%$ \\
\hline & & & & (59) Educación Primaria & 3 & $5,1 \%$ \\
\hline & & & & (38) Educación Social & 4 & $10,5 \%$ \\
\hline & \multirow{7}{*}{$\begin{array}{c}\text { (44) } \\
\text { Másteres }\end{array}$} & \multirow{7}{*}{$\begin{array}{c}\text { (7) } \\
\text { Másteres }\end{array}$} & \multirow{7}{*}{$15,9 \%$} & (12) Análisis histórico... & 2 & $16,6 \%$ \\
\hline & & & & (27) Estudios Ingleses... & 1 & $3,7 \%$ \\
\hline & & & & (18) Ed. Ambiental & 1 & $5,5 \%$ \\
\hline & & & & (26) Ed. Especial & 2 & $7,7 \%$ \\
\hline & & & & (24) Comunicación Social & 1 & $4,2 \%$ \\
\hline & & & & (25) Gestión Internac... & 2 & $8 \%$ \\
\hline & & & & (30) Migraciones.... & 8 & $26,6 \%$ \\
\hline \multirow{12}{*}{ Uై } & \multirow{3}{*}{$\begin{array}{c}(71) \\
\text { Grados }\end{array}$} & \multirow{3}{*}{$\begin{array}{c}\text { (3) } \\
\text { Grados }\end{array}$} & \multirow{3}{*}{$4,2 \%$} & (47) Educación Infantil & 1 & $2,1 \%$ \\
\hline & & & & (45) Trabajo Social & 4 & $8,8 \%$ \\
\hline & & & & (43) Psicología & 1 & $2,3 \%$ \\
\hline & \multirow{9}{*}{$\begin{array}{c}(59) \\
\text { Másteres }\end{array}$} & \multirow{9}{*}{$\begin{array}{c}(8) \\
\text { Másteres }\end{array}$} & \multirow{9}{*}{$13,5 \%$} & (12) Análisis histórico... & 2 & $16,6 \%$ \\
\hline & & & & (24) Comunicación Int... & 3 & $12,5 \%$ \\
\hline & & & & (25) Cultura de Paz... & 3 & $12 \%$ \\
\hline & & & & (18) Ed. Ambiental & 1 & $5,5 \%$ \\
\hline & & & & (12) Estudios Género... & 1 & $8,3 \%$ \\
\hline & & & & (22) Inv. Educativa... & 2 & $9,1 \%$ \\
\hline & & & & (10) Protección & 1 & $10 \%$ \\
\hline & & & & Jurídica... & 4 & $33,3 \%$ \\
\hline & & & & (12) Relaciones Inter... & & \\
\hline \multirow{7}{*}{ نَّ } & \multirow{3}{*}{$\begin{array}{c}(34) \\
\text { Grados }\end{array}$} & \multirow{3}{*}{$\begin{array}{c}(3) \\
\text { Grados }\end{array}$} & \multirow{3}{*}{$8,8 \%$} & (45) Educación Infantil & 1 & $2,2 \%$ \\
\hline & & & & (54) Educación Primaria & 3 & $5,5 \%$ \\
\hline & & & & (40) Educación Social & 5 & $12,5 \%$ \\
\hline & & & & (25) Cultura de Paz... & 3 & $12 \%$ \\
\hline & (58) & (4) & $60 \%$ & (18) Ed. Ambiental & 1 & $5,5 \%$ \\
\hline & Másteres & Másteres & $0,9 \%$ & (16) Enseñ. bilingüe... & 1 & $6,2 \%$ \\
\hline & & & & (28) Ed. Inclusiva & 5 & $17,8 \%$ \\
\hline & & & & (49) Educación Infantil & 1 & $2 \%$ \\
\hline & & & & (60) Antropología & 5 & $8 \%$ \\
\hline & $(102)$ & (5) & $40 \%$ & socia... & 2 & $4,2 \%$ \\
\hline & Grados & Grados & $4,9 \%$ & (47) Educación Social & 4 & $8,5 \%$ \\
\hline & & & & (47) Pedagogía & 3 & $6,5 \%$ \\
\hline & & & & (46) Trabajo Social & & \\
\hline & & & & (17) Artes visuales... & 1 & $5,9 \%$ \\
\hline & & & & (16) Cooperación des... & 1 & $6,2 \%$ \\
\hline$\frac{\pi}{0}$ & & & & (22) Diversidad & 9 & $41 \%$ \\
\hline$\Xi$ & & & & cultural... & 3 & $12 \%$ \\
\hline ڤँ & & & & (25) Cultura de Paz... & 1 & $5,5 \%$ \\
\hline & & & & (18) Ed. Ambiental & 1 & $7,1 \%$ \\
\hline & (112) & 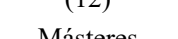 & $10,7 \%$ & (14) Interv. Psicoped... & 2 & $9,5 \%$ \\
\hline & & & & (21) Inv.. desarrollo... & 2 & $11,1 \%$ \\
\hline & & & & (18) Problemas soc... & 2 & $2,3 \%$ \\
\hline & & & & (86) Secundaria & 2 & $9,1 \%$ \\
\hline & & & & (22) Enseñ. Español... & 1 & $4,3 \%$ \\
\hline & & & & (23) Estudios latino.... & 1 & $4,3 \%$ \\
\hline & & & & (23) Lengua y culturas... & & \\
\hline & & & & (48) Educación Infantil & 2 & $4,2 \%$ \\
\hline & & & & (58) Educación Primaria & 1 & $1,7 \%$ \\
\hline & (3/) & (5) & $13,5 \%$ & (52) Educación Social & 6 & $11,5 \%$ \\
\hline 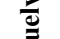 & & & & (49) Psicología & 1 & $2 \%$ \\
\hline E & & & & (46) Trabajo Social & 4 & $8,7 \%$ \\
\hline & (38) & (6) & $15.70 /$ & (18) Ed. Ambiental & 1 & $5,5 \%$ \\
\hline & Másteres & Másteres & $10,7>0$ & (14) Comunicación y & 1 & $7,1 \%$ \\
\hline
\end{tabular}




\begin{tabular}{|c|c|c|c|c|c|c|}
\hline & & & & Ed... & 2 & $11,1 \%$ \\
\hline & & & & (18) Ed. Especial & 3 & $17,6 \%$ \\
\hline & & & & (17) Innovación... & 1 & $5,3 \%$ \\
\hline & & & & (19) Inv. e Interv... & 1 & $3,3 \%$ \\
\hline & & & & (30) Secundaria & & \\
\hline \multirow{3}{*}{ 麦 } & \multirow{3}{*}{$\begin{array}{c}\text { (20) } \\
\text { Másteres }\end{array}$} & \multirow{3}{*}{$\begin{array}{c}(2) \\
\text { Másteres }\end{array}$} & \multirow[b]{3}{*}{$10 \%$} & (14) Comunicación y & 1 & $7,1 \%$ \\
\hline & & & & Ed... & 2 & $7,1 \%$ \\
\hline & & & & (28) Derechos human... & & \\
\hline \multirow{6}{*}{ "ِّ } & \multirow{4}{*}{$\begin{array}{c}(50) \\
\text { Grados }\end{array}$} & \multirow{4}{*}{$\begin{array}{c}(4) \\
\text { Grados }\end{array}$} & \multirow{4}{*}{$8 \%$} & (65) Educación Infantil & 1 & $1,5 \%$ \\
\hline & & & & (65) Educación Primaria & 2 & $3,2 \%$ \\
\hline & & & & (42) Educación Social & 1 & $2,4 \%$ \\
\hline & & & & (49) Trabajo Social & 3 & $6,1 \%$ \\
\hline & \multirow{2}{*}{$\begin{array}{c}\text { (53) } \\
\text { Másteres }\end{array}$} & (2) & \multirow{2}{*}{$3,8 \%$} & (12) Análisis histórico... & 2 & $16,6 \%$ \\
\hline & & Másteres & & (16) Dependencia e ig... & 1 & $6,25 \%$ \\
\hline \multirow{12}{*}{$\frac{\sqrt{50}}{\frac{\pi}{\pi / \pi}}$} & \multirow{5}{*}{$\begin{array}{c}(75) \\
\text { Grados }\end{array}$} & \multirow{5}{*}{$\begin{array}{c}(5) \\
\text { Grados }\end{array}$} & \multirow{5}{*}{$6,6 \%$} & (43) Educación Infantil & 2 & $4,6 \%$ \\
\hline & & & & (81) Educación Primaria & 3 & $3,7 \%$ \\
\hline & & & & (44) Educación Social & 3 & $6,8 \%$ \\
\hline & & & & (43) Pedagogía & 3 & $7 \%$ \\
\hline & & & & (49) Trabajo Social & 5 & $10,2 \%$ \\
\hline & \multirow{7}{*}{$\begin{array}{c}\text { (72) } \\
\text { Másteres }\end{array}$} & \multirow{7}{*}{$\begin{array}{c}\text { (7) } \\
\text { Másteres }\end{array}$} & \multirow{7}{*}{$9,7 \%$} & (25) Cultura de Paz... & 3 & $12 \%$ \\
\hline & & & & (18) Ed. Ambiental & 1 & $5,5 \%$ \\
\hline & & & & (14) Cambio Social... & 1 & $7,1 \%$ \\
\hline & & & & (18) Inv. e interv... & 2 & $11,1 \%$ \\
\hline & & & & (19) Mediación & 2 & $10,5 \%$ \\
\hline & & & & (31) Psicopedagogía & 1 & $3,2 \%$ \\
\hline & & & & (23) Secund.+Est. Ing... & 2 & $8,7 \%$ \\
\hline \multirow{10}{*}{ 잉 } & \multirow{5}{*}{$\begin{array}{c}(44) \\
\text { Grados }\end{array}$} & \multirow{5}{*}{$\begin{array}{c}(5) \\
\text { Grados }\end{array}$} & \multirow{5}{*}{$11,3 \%$} & (60) Comunicación & 1 & $1,6 \%$ \\
\hline & & & & (43) Comunicación D... & 1 & $2,3 \%$ \\
\hline & & & & (40) Educación Social & 6 & $15 \%$ \\
\hline & & & & (40) Sociología & 3 & $7,5 \%$ \\
\hline & & & & (49) Trabajo Social & 5 & $10,2 \%$ \\
\hline & & & & (12) Análisis histórico... & 2 & $16,6 \%$ \\
\hline & & (5) & & (18) Ed. Ambiental & 1 & $5,5 \%$ \\
\hline & Másteres & Másteres & $12,2 \%$ & (28) Derechos human... & 2 & $7,1 \%$ \\
\hline & & & & (24) Ed. Desarrollo... & 1 & $4,2 \%$ \\
\hline & & & & (24) Interv. Social... & 1 & $4,2 \%$ \\
\hline & & & & (42) Educación Infantil & 1 & $2,4 \%$ \\
\hline & (67) & (4) & & (48) Educación Primaria & 2 & $4,2 \%$ \\
\hline & Grados & Grados & $6 \%$ & (45) Pedagogía & 4 & $8,8 \%$ \\
\hline & & & & (38) Antropología & 5 & $13,2 \%$ \\
\hline & & & & socia... & & \\
\hline & & & & (48) Secund.+Est.Ling... & 1 & $2,1 \%$ \\
\hline$\overline{2}$ & & & & (15) Estudios Género... & 1 & $6,6 \%$ \\
\hline & & & & (31) Psicopedagogía & 1 & $3,2 \%$ \\
\hline & (88) & (8) & $91 \%$ & (15) Comunicación y c... & 1 & $6,6 \%$ \\
\hline & Másteres & Másteres & $8.1 \%$ & (17) Antropología... & 4 & $23,5 \%$ \\
\hline & & & & (20) Psicología ed... & 1 & $5 \%$ \\
\hline & & & & (19) Psicología interv... & 1 & $5,2 \%$ \\
\hline & & & & (13) Migraciones... & 8 & $61,5 \%$ \\
\hline
\end{tabular}

Tabla 1. Interculturalidad en Universidades y Titulaciones Oficiales de Andalucía

A partir de aquí, se realiza un muestreo intencional en el que se seleccionan diversas Universidades basándose en las variables que siguen a continuación:

a) Datos significativos en el saldo migratorio (positivo o negativo).

b) Cantidad de menciones en el currículo universitario relativas a la Educación Intercultural. 
En relación a la primera, es preciso identificar que el saldo migratorio viene a ser la resta del total de inmigraciones menos las emigraciones. A partir de aquí, en Andalucía, según los datos del Instituto de Estadística y Cartografía de Andalucía (Junta de Andalucía, 2020), durante el año 2019 los movimientos migratorios ofrecieron un saldo positivo, confirmando una tendencia en alza; en dicho año se obtuvieron 168.277 entradas y 109.803 salidas. Las provincias de Málaga, Almería y Huelva, fueron de las que más entradas recibieron desde el extranjero y Jaén fue la que menos entradas recibió; por tanto, Málaga presentó el mayor saldo positivo (25.727) y Jaén el mayor saldo migratorio negativo (-1.093).

Basándose en la segunda variable, la frecuencia de aparición de la Educación Intercultural da como resultado el siguiente ranking: Universidades de Huelva (29,2\%), Almería (24,7\%), UPO (23,5\%), Cádiz (17,7\%), Málaga (16,3\%), Córdoba (15,7\%), Granada (15,6\%), Jaén (11,8\%) y UNIA (10\%).

Así pues, si atendemos a la triangulación de los datos obtenidos en el estudio de estas dos variables, obtenemos que la Universidad cuya provincia tuvo mayor saldo migratorio fue Málaga y la que obtuvo un saldo negativo fue Jaén; y, por otra parte, se localizan las universidades de Huelva y de Almería como las que poseen mayor referencia en sus planes de estudios hacia la Educación Intercultural. Se consideran así estas 4 universidades como objetos de estudio.

\subsection{Instrumento}

Para la recuperación de la información clave procedente de las guías docentes y planes de estudios, se diseñó una plantilla de registro (Tabla 2).

\begin{tabular}{lll}
\hline Universidad & $\begin{array}{l}\text { Titulación } \\
\text { (grado/máster) }\end{array} \quad$ Asignaturas & $\begin{array}{l}\text { Frecuencia de aparición de descriptores clave (en } \\
\text { bloques de objetivos, contenidos o competencias) }\end{array}$ \\
\hline
\end{tabular}

Tabla 2. Hoja de registro de información

Se trata de un registro de observación diseñado ad hoc. Este instrumento tiene como fundamento de partida ayudar a organizar la información mediante la localización, en los textos de referencia, de aquellos términos que son afines a lo que implica la Educación Intercultural. En concreto, se entendieron como descriptores: migraciones, Educación Intercultural, cultura, multiculturalidad, interculturalidad, emigración, saldo migratorio, aculturación, transculturación, etnocentrismo, derechos humanos, etnografía, identidad, estratificación social, diversidad cultural, ética, mediación intercultural, antropología.

\subsection{Procedimiento y análisis de la información}

La investigación se desarrolló durante el curso 2019/2020 y su carácter analítico hace que se desarrolle de forma longitudinal, pero acotando el periodo de estudio a un curso académico para evitar posibles modificaciones en las guías o planes de estudios. Se trata de un procedimiento no interactivo, en el que el análisis documental se desarrolla a través de la visita a las páginas webs de las Universidades Andaluzas y los correspondientes espacios virtuales que registran las titulaciones y el material de 
referencia (planes de estudios y guías docentes de títulos de grados y másteres oficiales).

Una vez se localizan los documentos objeto del análisis, se procede a la búsqueda selectiva en ellos mediante el empleo de los descriptores claves mencionados anteriormente. Basándose en la aparición de dichos conceptos (asumiendo el criterio de saturación de información), se toma la decisión de considerar que en un documento se recoge información suficiente como para catalogarlo como favorecedor de la Educación Intercultural. Una vez identificados los documentos idóneos para el estudio, y secuenciada la información derivada de este primer filtro de análisis, se descargan y se almacenan para continuar con su análisis en profundidad.

El segundo paso, se apoya en el tratamiento de la información a partir del software MAXQDA v.10. Se establece un sistema de categorías basándose en las diferentes ramas de conocimiento científico asociadas al campo de las Ciencias Humanas y Sociales y sus correspondientes códigos:

- Categorías y códigos: "Pedagogía", con el código "intervención educativa"; "Antropología", con los códigos "cultura" y "etnografía"; "Filosofía", con los códigos "fines" y "comportamientos éticos"; "Sociología", con los códigos "estratificación social" e "interacción social"; "Psicología", con el código "identidad"; y "Derecho", con los códigos "derechos humanos" y "delitos de odio".

Todo ello para clasificar y segmentar la información procedente de la documentación de referencia. Además, este programa ha permitido también realizar procedimientos de conteo de palabras, obtención de frecuencias o generación de mapas conceptuales que facilitan perfilar los procesos de codificación desarrollados y descubrir patrones de interés entre la información recopilada.

\section{RESULTADOS}

Una vez codificada toda la información procedente del análisis documental, a través del MAXQDA se diseña una nube de conceptos (Figura 2) mediante la que se visualizan con claridad los principales términos que, ligados a la Educación Intercultural, se recogen en las guías docentes y planes de estudios analizados. En este sentido, las principales frecuencias de palabras señalan que conceptos como la propia Educación Intercultural, saldo migratorio, diversidad cultural, cultura, inmigración, interculturalidad o multiculturalidad, son los más repetidos en la enseñanza superior, cuando esta trata de potenciar el uso de contenido ligado al marco de la Educación Intercultural en la formación de los futuros profesionales. Así pues, este recurso visual ha de servir para disponer de una primera impresión relativa al tratamiento del objeto de estudio en este tipo de documentos. 


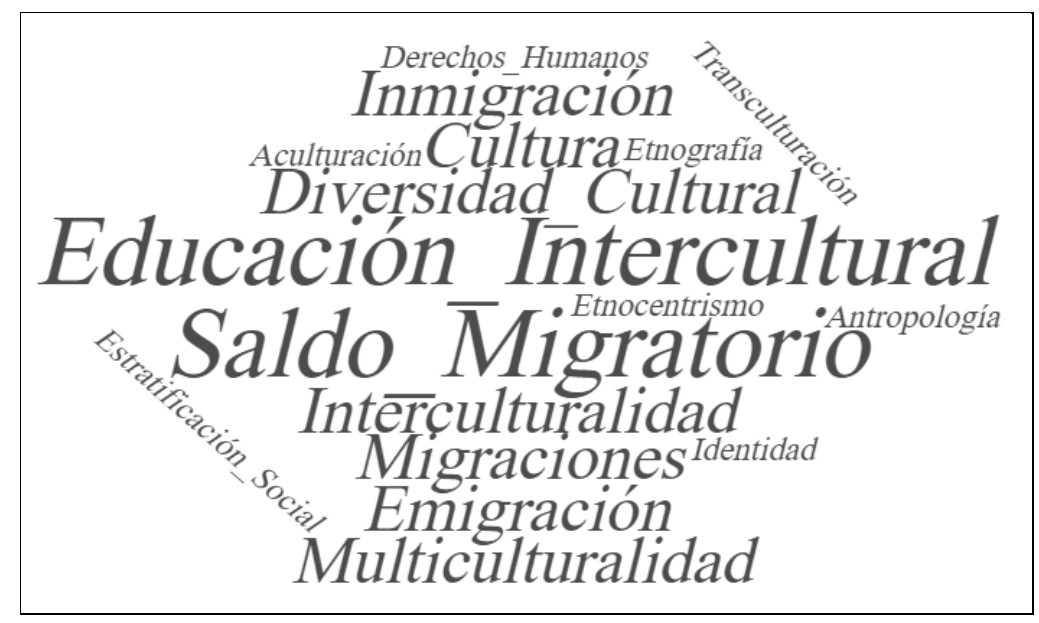

Figura 2. Nube de palabras resultante del análisis de frecuencias

Además de esta representación visual, los resultados obtenidos en este estudio profundizan en las realidades existentes en cada una de la universidades analizadas. A continuación, se presenta una tabla descriptiva (Tabla 3):

\begin{tabular}{|c|c|}
\hline Universidad & Área de Conocimiento \\
\hline \multirow{8}{*}{ Almería } & Pedagogía: Grado en Educación Social (10,5\%). \\
\hline & Máster en Mediación y Grupos vulnerables $(26,6 \%)$ \\
\hline & Antropología: Máster en Migraciones, Mediación y Grupos Vulnerables \\
\hline & $(26,6 \%)$ \\
\hline & Filosofía: Máster en Comunicación Social (4,2\%) \\
\hline & Sociología: Máster de Análisis Histórico del Mundo Actual (16,6\%) \\
\hline & Psicología: sin mención a la interculturalidad \\
\hline & Derecho: Máster en Gestión Internacional de la Empresa e Idiomas (8\%) \\
\hline \multirow{7}{*}{ Huelva } & Pedagogía: Grado en Educación Social $(11,5 \%)$ \\
\hline & Máster en Innovación y Liderazgo Educativo (17,6\%) \\
\hline & $\begin{array}{l}\text { Antropología: Master en Investigación e Intervención en Trabajo Social } \\
(5,3 \%)\end{array}$ \\
\hline & Filosofía: sin mención a la Educación Intercultural \\
\hline & Sociología: Grado en Trabajo Social $(8,7 \%)$ \\
\hline & Psicología: Grado en Psicología (2\%) \\
\hline & Derecho: sin mención a la Educación Intercultural \\
\hline \multirow{7}{*}{ Jaén } & Pedagogía: Grado en Educación Social $(2,4 \%)$ \\
\hline & Antropología: sin mención a la Educación Intercultural \\
\hline & Filosofía: sin mención a la Educación Intercultural \\
\hline & Sociología: Grado en Trabajo Social $(6,1 \%)$ \\
\hline & Máster de Análisis Histórico del Mundo Actual $(16,6 \%)$ \\
\hline & Psicología: sin mención a la Educación Intercultural \\
\hline & Derecho: sin mención a la Educación Intercultural \\
\hline \multirow{7}{*}{ Málaga } & Pedagogía: Grado en Pedagogía (7\%) \\
\hline & Máster en cultura de paz, conflictos, educación y derechos humanos $(12 \%)$ \\
\hline & Antropología: sin mención a la Educación Intercultural \\
\hline & Filosofía: sin mención a la Educación Intercultural \\
\hline & Sociología: Grado en Trabajo Social $(10,2 \%)$ \\
\hline & Psicología: Máster en Psicopedagogía (3,2\%) \\
\hline & Derecho: Máster de Mediación (10,5\%) \\
\hline
\end{tabular}

Tabla 3. Presencia de la Educación Intercultural en universidades y áreas de conocimiento

Atendiendo a la codificación de las universidades, basándose en cada una de las áreas de conocimiento, se puede observar que en la categoría de Pedagogía, la Universidad de 
Almería tiene el porcentaje más elevado de titulaciones con mención de Educación Intercultural, siendo el porcentaje más elevado el del Máster en Mediación y Grupos Vulnerables (26,6\%). En cambio, en cuanto a los Grados, es en la Universidad de Huelva donde reside el porcentaje más alto, en Educación Social, con un 11,5\%.

En cuanto a la categoría de Antropología, resaltar que es la Universidad de Almería también la que tiene el mayor porcentaje en este sentido, en el Máster en Migraciones, Mediación y Grupos Vulnerables con un 26,6\%.

En la categoría de Filosofía, de nuevo resaltar a la Universidad de Almería con el Máster en Comunicación Social, pero en este caso con solo un 4,2\%.

En referencia a la categoría Sociología, reseñar que la Universidad de Almería con el Máster de Análisis Histórico del Mundo Actual (16,6\%) y la Universidad de Málaga con el Grado en Trabajo Social (10'2\%), son en las que mayor número de asignaturas poseen una mención relativa a la Educación Intercultural.

La categoría de Psicología encuentra una mayor mención en Educación Intercultural en el Máster en Psicopedagogía de la Universidad de Málaga (3,2\%); por su parte, en la Universidad de Huelva, es el Grado de Psicología donde existe un 2\% de mención a la Educación Intercultural.

Por último, en la categoría Derecho, es la Universidad de Málaga la que tiene mejor mención a la Educación Intercultural con el Máster de Mediación (10,5\%).

\section{CONCLUSIONES}

Las enseñanzas en Educación Intercultural han ido cambiando con la aparición de las nuevas titulaciones de Grado y de Máster Oficial en nuestra sociedad. Éstas se introdujeron poco a poco en estudios sobre migraciones, siendo hoy en día un requisito imprescindible para llevar a cabo ciertas actividades de enseñanza. Tanto es así, que, desde hace unos años, son más numerosas las asignaturas especializadas que se enfocan en la Educación Intercultural.

Estas titulaciones oficiales se encuentran ubicadas en las páginas webs de cada una de las universidades analizadas, donde se pueden visualizar cada una de las guías docentes (fácilmente localizables y accesibles) con una serie de características peculiares (objetivos, contenidos, competencias, etc.) y con cierta predilección por las enseñanzas de índole pedagógico como gran reclamo.

Por otro lado, en cada una de las titulaciones se priorizan asignaturas de una determinada profesión, convirtiéndose en un hándicap a la hora de elegir un tipo de enseñanza u otra.

Por todo ello, se enumera una serie de propuestas de mejora para que la enseñanza de la Educación Intercultural aumente en las guías docentes y en los planes de estudio:

- Para las Titulaciones Oficiales: focalizar más las asignaturas de contenidos ligados a la Educación Intercultural a los Grados, ya que existen Másteres específicos que se dedican en buena parte a ello, por tanto, es conveniente dotar de más créditos a la enseñanza más generalista, para compensarla con la enseñanza más especializada.

- Para las Universidades: tener en cuenta los datos publicados en el Instituto de Estadística y Cartografía de Andalucía (2020), para fomentar más enseñanzas sobre Educación Intercultural en aquellas provincias donde más se produzca ese 
saldo migratorio positivo. Un ejemplo es el caso de Málaga, donde existe el mayor saldo migratorio positivo, pero donde apenas destacan titulaciones con carácter intercultural.

- Para las Guías Docentes: es de utilidad reflejar cada uno de los apartados como aparecen, pero carecen de detalles específicos sobre la Educación Intercultural, salvo que el nombre de la asignatura lo especifique.

- Para las Titulaciones con mención a la Educación Intercultural: es conveniente fomentar la introducción de más asignaturas de esta índole, para poder trabajar uno de los objetivos generales de la educación, como es fomentar la diversidad cultural en todos los ámbitos de la sociedad.

En definitiva, los principios y las prácticas asociadas a la enseñanza tradicional siguen siendo algo sumamente evidente en los currículos de las titulaciones oficiales actuales; en gran medida, ello está motivado por la incorporación de diferentes asignaturas básicas (tras la llegada del Espacio Europeo de Educación Superior), cuyos contenidos acentúan la discrepancia en la opinión del alumnado a la hora de valorar la significatividad de los procesos de enseñanza y de aprendizaje existentes. La triangulación, por tanto, entre el profesorado, el alumnado y las titulaciones debe perseguir una serie de objetivos generales que fomenten la enseñanza de la Educación Intercultural, como uno de los aspectos básicos de toda titulación oficial y que ayuden a mejorar la calidad de las enseñanzas regladas.

\section{BIBLIOGRAFÍA}

Aguado, M.T. (1991). La Educación Intercultural: concepto, paradigmas y realizaciones. En C. Jiménez (ed.). Lecturas de Pedagogía Diferencial (pp. 87-104). Dykinson.

Bernabé, M.M. (2016). Formación para la ciudadanía intercultural en Educación Infantil. ¿Qué sucede en las universidades valencianas? Revista de currículum y formación del profesorado, 20(2), 328-348. Recuperado de: https://n9.cl/1n7qn

Bueno, J.J. (2008). Nuevos retos, nuevas perspectivas para la Educación multicultural. Revista Educación Inclusiva, 1, 59-76. Recuperado de: https://n9.cl/8ij2i

Cala, V.C., Soriano-Ayala, E. y López-Martínez, M.J. (2018). Actitudes hacia personas refugiadas y ciudadanía europea inclusiva. Análisis para una propuesta educativa intercultural con el profesorado en formación. RELIEVE, 24(2), art. M1. https://doi.org/10.7203/relieve.24.2.13320

Carbonell, F. (1995). Inmigración, diversidad cultural, desigualdad social y educación. MEC.

Carrasco, M.J. y Coronel, J.M. (2017). Percepciones del profesorado sobre la gestión de la diversidad cultural: Un estudio cualitativo. Educación XX1, 20(1), 75-98. https://doi.org/10.5944/educxx1.17492

Díaz De Rada, A. (2010). Cultura, antropología y otras tonterías. Editorial Trotta.

Di Caudo-Villoslada, M.V. (2020). "No estamos de acuerdo con el título". Escribir un libro con estudiantes universitarios interculturales. Magis, 13, 1-27. https://doi.org/10.11144/Javeriana.m13.neat

Etxeberría, F. y Elósegui, C. (2010). Integración del alumnado inmigrante: obstáculos y propuestas. Revista Española de Educación Comparada, 16, 235-263. Recuperado de: https://n9.cl/rl7i7

Espinoza, E., Herrera, L.A. y Castellano, J.M. (2019). La dimensión intercultural en la formación docente en Ecuador. Psicología, Sociedad y Educación, 11(3), 341-354. https://doi.org/10.25115/psye.v11i3.2253 
Fernández-Agüero, M. y Garrote, M. (2019). 'No es mi competencia intercultural, soy yo.' La identidad intercultural del profesorado de lenguas extranjeras en formación. Educar, 55(1), 159-182. https://doi.org/10.5565/rev/educar.985

Gallardo, H. (2016). Teoría crítica y derechos humanos. Una lectura latinoamericana. Colectivo sobre Teoría Crítica y derechos humanos.

García, F.J., Barragán, C. y Granados, A. (2004). Multiculturalismo, etnoeducación y Educación Intercultural. Memorias del Primer Foro de Etnoeducación Afrocolombiana. Ministerio de Educación Nacional.

García Llamas, J.L. (2005). Educación Intercultural. Análisis y propuestas. Revista de Educación, 336, 89-109. Recuperado de: https://n9.cl/ze3f1

Gil-Madrona, P., Gómez-Barreto, I. y González-Víllora, S. (2016). Percepción de los estudiantes de maestro de educación infantil sobre su formación intercultural. Magis, 9, 111-128. https://doi.org/10.11144/Javeriana.m9-18.pmei

Giménez, C. (2003). Pluralismo, Multiculturalismo e Interculturalidad. Propuesta de clarificación y apuntes educativos. Educación y Futuro digital: Educación Intercultural, 8, 9-26. Recuperado de: https://n9.cl/ajlxo

González-Falcón, I. (2003). La inmigración en España. Un nuevo reto social y educativo (Trabajo de Investigación. Bienio de Doctorado 2001-2003). Universidad de Huelva. Inédito.

González-Falcón, I. (2006). Formación del profesorado, inmigración e interculturalidad. Reflexiones desde la escuela. El Guiniguada, 15-16, 95-108. Recuperado de: https://n9.cl/bmthy

González-González, H., Álvarez-Castillo, J.L. y Fernández-Caminero, G. (2012). Fundamentando la formación de los educadores profesionales en competencias interculturales: la toma de perspectiva. Bordón, 64(1), 89-107. Recuperado de: https://n9.cl/i9x3w

Grañeras, M., Lamelas, R., Segalerva, A., Vázquez, E., Gordo, J.L. y Molinuevo, J. (1998). La investigación sobre Educación Intercultural. En M. Grañeras, R. Lamelas, A. Segalerva, E. Vázquez, J.L. Gordo y J. Molinuevo (Eds). Catorce años de investigación sobre las desigualdades sociales en España (pp. 111-150). CIDE.

Han, B. (2018). Hiperculturalidad. Herder Editorial.

Herrera, J. (2005). Prólogo al libro de Boaventura de Sousa Santos. Foro Social Mundial. Manual de uso. Icaria.

Hidalgo, V. (2005). Cultura, multiculturalidad, interculturalidad y transculturalidad: Evolución de un término. Universitas tarraconensis: Revista de ciències de l'educació, 1, 75-85. Recuperado de: https://n9.cl/etsm7

Horkheimer, M. (2000). Teoría tradicional y teoría crítica. Ediciones Paidós.

Junta de Andalucía (2020). Estadística de variaciones residenciales. Andalucía: Instituto de Estadística y Cartografía de Andalucía. Recuperado de: https://cutt.ly/KtiD9vP

Louzao, M. (2019). Educación Intercultural: de la urgencia cuantitativa a la exigencia democrática. Orientaciones para la investigación y la acción en el contexto educativo asturiano. Revista de Investigación en Educación, 17(1), 44-54. Recuperado de: http://reined.webs.uvigo.es/index.php/reined/article/view/378

Lozano, R. (2005). Interculturalidad: Desafío y proceso en construcción. Manual de capacitación. Servicios en Comunicación Intercultural-SERVINDI. Sinco Editores.

McMillan, J.H. y Schumacher, S. (2005). Investigación Educativa. Pearson Editorial S.A.

Molina, J.F. (1994). Sociedad y educación: perspectivas interculturales. Universidad de Lleida y Promociones y Publicaciones Universitarias.

OIM (2006). Glosario sobre migración. Derecho Internacional sobre migración. Organización Internacional para las migraciones.

Peñalva, A. y Leiva, J.J. (2019). La interculturalidad en el contexto universitario: necesidades en la formación inicial de los futuros profesionales de la educación. Educar, 55(1), 141-158. https://doi.org/10.5565/rev/educar.989 
Popkewitz, T.S., Pitman, A. y Barry, A. (1986). Educational reform and its millennial quality: the 1980s. Journal of Curriculum Studies, 18(3), 267-283. https://doi.org/10.1080/0022027860180304

Puig, J.M. (1995). La educación moral en la enseñanza obligatoria. Horsori Editoral.

Sáez-López, J.M., Miyata, Y. y Domínguez-Garrido, M.C. (2016). Codificación creativa y proyectos interculturales en Educación Superior: Un estudio de caso en tres universidades. RIED, 19(2), 145-165. https://doi.org/10.5944/ried.19.2.15796

Salvat, P. (2001). Universalismo y contextualismo: ¿es posible y/o necesaria una ética universalista hoy? Revista Novamérica, 91, 31. Recuperado de: https://www.yumpu.com/es/document/view/13725295/universalismo-y-contextualismouniversidad-alberto-hurtado

Soltonovich, A. (2012). Derechos humanos y teoría crítica de la sociedad. En M.H. Respreto (Coord.). Derechos Humanos en tiempos de globalización y neoparamilitarismo en Colombia. UPTC.

Touriñán López, J.M. (2005). Educación en valores, educación intercultural y formación para la convivencia pacífica. Revista Galega do Ensino, 47, 1.367-1.418. Recuperado de: https://cutt.ly/jRlKbme

Walsh, C. (2007). Interculturalidad, colonialidad y educación. Revista Educación y Pedagogía, 19(48), 25-35. Recuperado de: https://n9.cl/a436v 